\title{
Utility of 3D SPACE T2-weighted volumetric sequence in the localization of spinal dural arteriovenous fistula
}

\author{
Santhosh Kumar Kannath, MD, PDCC, ${ }^{1}$ Praveen Alampath, DNB, DM, ${ }^{1}$ \\ Jayadevan Enakshy Rajan, MD, DM, ${ }^{1}$ Bejoy Thomas, MD, PDCC, ${ }^{1}$ \\ P. Sankara Sarma, MSc, MHS, PhD, ${ }^{2}$ and Kapilamoorthy Tirur Raman, MD, PDCC ${ }^{1}$ \\ 'Department of Imaging Sciences and Interventional Radiology, and ${ }^{2}$ Achutha Menon Centre for Health Science Studies, \\ Sree Chitra Institute for Medical Sciences and Technology, Trivandrum, India
}

OBJECTIVE The aim of this study was to investigate the utility of a heavily T2-weighted volumetric MRI sequence (3D sampling perfection with application-optimized contrasts using different flip-angle evolutions [SPACE]) in the feeder localization of spinal dural arteriovenous fistula (SDAVF).

METHODS Patients who were diagnosed with SDAVF and who had 3D SPACE source images available for review were identified from a retrospective review of medical records. A total of 16 patients were identified, and MR images were analyzed separately by 2 blinded observers. The accuracy of the observation and interobserver agreement were measured by Kendall's tau and kappa statistics.

RESULTS The site of fistula was accurately predicted by Observers 1 and 2 in $81 \%$ and $88 \%$ of cases, respectively, which improved to $94 \%$ when the level was considered within 1 vertebral level. The observer agreement with gold-standard angiography and interobserver agreement were found to be highly significant $(p<0.0001)$.

CONCLUSIONS The 3D SPACE MRI sequence is valuable in the precise detection of the site of SDAVF. It may help to tailor digital subtraction angiography and thereby reduce the radiation exposure, contrast load, and study time.

http://thejns.org/doi/abs/10.3171/2015.8.SPINE141322

KEY WORDS spinal dural fistula; high-resolution volumetric sequence; localization; sampling perfection with application-optimized contrasts using different flip-angle evolutions (SPACE); vascular disorders

$\mathrm{S}$ PINAL dural arteriovenous fistula (SDAVF) is the most common vascular malformation of the spine. It is characterized by fistulous communication of radiculomeningeal artery and radicular vein on the dural sleeve of the dorsal root ganglion. Although SDAVF accounts for $60 \%-80 \%$ of all vascular malformations of the spine, the disease is rare and usually remains undiagnosed for a significant period due to nonspecific clinical symptoms. ${ }^{10,12}$ It is often neuroradiological imaging that raises the suspicion of dural fistula; however, confirmation of the diagnosis requires invasive spinal angiographic study. MRI findings such as cord signal abnormalities and flow voids are highly suggestive of SDAVF and warrant further evaluation with spinal angiography. ${ }^{22}$

Spinal angiography remains the gold standard for the evaluation and characterization of SDAVF. It additionally offers the possibility of endovascular obliteration of the fistula, which is increasingly being considered as the initial therapeutic option, with surgery reserved for situations such as failed embolization or unfavorable anatomy. ${ }^{11}$ Spinal angiography involves selective angiographic evaluation of the segmental arteries from the cervical level to the sacrum. The procedure is relatively straightforward with few complications. However, it may become time-consuming,

ABBREVIATIONS CTA = CT angiography; DSA = digital subtraction angiography; FOV = field of view; MIP = minimal-intensity projection; MRA = MR angiography; SDAVF = spinal dural arteriovenous fistula; SPACE = sampling perfection with application-optimized contrasts using different flip-angle evolutions, $\mathrm{T} 2 \mathrm{~W}=\mathrm{T} 2$-weighted. SUBMITTED April 1, 2015. ACCEPTED August 24, 2015. 
especially if the aorta is atherosclerotic and exposes the patient to higher radiation exposure and contrast overload. ${ }^{8}$ Noninvasive imaging methods, such as contrast-enhanced MR angiography (MRA) and CT angiography (CTA), have been shown to have higher sensitivity and specificity in the detection and prediction of the location of the fistula. 1,2,14,17,19,23 Higher magnet strengths, faster imaging techniques due to higher gradient performances, larger field of view (FOV), and robust data-sampling methods have improved the diagnostic performance of MRI. However, such technical specifications may not be available for all MR machines. ${ }^{15,17}$ Additionally, the patient is exposed to the risk of nephrogenic systemic fibrosis, especially when the renal parameters may be deranged in situations such as long-standing bladder symptoms and hydronephrosis.

Recently, a high-resolution T2-weighted volumetric sequence $(3 \mathrm{D}$ sampling perfection with applicationoptimized contrasts using different flip-angle evolutions [SPACE]) was introduced. A 3D SPACE sequence acquires isotropic volumetric data that can be reconstructed in any plane with good T2 contrast and acceptable examination times. ${ }^{3}$ The sequence provides excellent tissue contrast between the cord parenchyma and CSF. Also, CSF flow artifacts that occasionally mimic flow voids in other routine sequences are suppressed in this sequence and thus improve the diagnostic confidence, especially when the changes are subtle. These advantages, combined with submillimeter voxel resolution, may distinctively demonstrate the abnormal spinal vascular channels. In the present study, we sought to evaluate the potential usefulness of 3D SPACE in identifying the location of fistula in patients with SDAVF that was confirmed with gold-standard spinal digital subtraction angiography (DSA).

\section{Methods}

A retrospective review of institutional records was performed. All patients who were diagnosed with SDAVF by gold-standard spinal angiographic study during the period from January 2007 to January 2013 were selected. Spinal vascular malformations, such as spinal cord arteriovenous malformations, perimedullary fistulas, and cavernomas were excluded from the analysis. Patients were evaluated to confirm the availability of conventional MRI and volumetric 3D SPACE T2-weighted sequences performed at our institution and were subsequently recruited for the study.

Subjects were excluded if the spinal DSA and MR images were of suboptimal interpretable quality or were incomplete. The DSA images were considered optimal if the angiographic studies of vertebral, subclavian, superior intercostal, intercostal, lumbar, and sacral arteries were obtained and were of diagnosable quality. The MR images were considered optimal if they were free of artifacts and the region of interest was adequately covered in all of the sequences. Specifically, in 3D SPACE sequences, images were scrutinized for inclusion of lateral edges of vertebrae and adjacent paravertebral spaces to ensure adequate evaluation of the neural foramen and neurovascular structures.

Patients were also excluded if the interval between MRI and DSA exceeded more than 2 weeks. The MR images were scrutinized for interpretability and were read by 2 interventional neuroradiologists with more than 5 and 2 years of experience, respectively, who were blinded to the angiographic diagnosis. The study received institutional ethics committee approval.

All patients underwent MRI with a 1.5-T superconducting MR system (Avanto SQ-engine, Siemens). The MRI protocol included the 3D volumetric T2-weighted SPACE sequence, in addition to the routine sequences such as T1- and T2-weighted sagittal, T1- and T2-weighted axial, and gradient T2-weighted sagittal sequences. The imaging parameters of the SPACE sequence were uniform during the study period and were as follows: TR/TE, 1500 msec/143 msec; number of excitations, 2; FOV, 280; flip angle, $150^{\circ}$; slab thickness, $0.9 \mathrm{~mm}$; matrix, $370 \times 320$; reconstructed voxel size, $1 \times 1 \times 1 \mathrm{~mm}$; and total time of acquisition, 10.26 minutes. This sequence was performed in 2 stages covering cervicothoracic and thoracolumbosacral regions, from which whole-spine images were created using proprietary fusion software provided by the MR vendor. The MR study protocol remained the same throughout the study period. These images were analyzed in a PACS (picture archiving and communication system) (AGFA Healthcare NV) in multiple planes as well as in VOXAR 3D software installed in the PACS. Spinal angiography was performed via a femoral route following a standard technique described elsewhere. ${ }^{6}$

The 3D SPACE volumetric images were initially reviewed for the presence of cord signal changes and flow voids. The images were scrolled to either side and the level of maximal venous congestion was identified. This was determined by noting the level of maximum cluster of veins in the spinal subarachnoid space. From this level, the images were scrolled to identify the presence of a single vein that may have arisen from the congested venous plexus, and it was followed rostrally or caudally until it coursed close to the pedicle of a particular vertebral body. At this level, axial reconstruction was reviewed and the course of this vein close to the pedicle was confirmed. The pedicle that it was closely related to, beyond which it was not traceable or beyond which it indeed exited through the neural foramina and communicated with the dorsal branch of the intercostal artery, was considered the feeder to the SDAVF. In situations where the vein could not be followed due to excess tortuosity, it was traced in multiple planar reconstructions and the level was identified.

The single vein was thought to correspond to the radicular vein, which refluxed into the congested perimedullary veins identified as the plexus of veins in MRI. The pedicle to which it was traced was hypothesized to be the location of the dural fistula, and the radicular artery supplying the foramen was considered the feeder of the fistula. The interpreters analyzing the MR images were blinded to the angiographic findings, which were correlated with the gold-standard spinal angiographic study. The accuracy of localization and interobserver agreements were calculated by Kendall's tau and the side of fistula was assessed by kappa statistics.

\section{Results}

A retrospective review identified 16 patients with SDAVF who met the inclusion criteria during the study pe- 
TABLE 1. Clinical and demographic data of 16 patients with SDAVF included in the study

\begin{tabular}{|c|c|c|c|c|}
\hline $\begin{array}{l}\text { Case } \\
\text { No. }\end{array}$ & $\begin{array}{l}\text { Age, } \\
\text { Yrs }\end{array}$ & Sex & Presentation & Duration \\
\hline \multirow[t]{3}{*}{1} & \multirow[t]{3}{*}{46} & \multirow[t]{3}{*}{ M } & Bilat LL weakness & 3 mos \\
\hline & & & LL paresthesia & $3 \mathrm{mos}$ \\
\hline & & & Urinary retention \& constipation & $1 \mathrm{wk}$ \\
\hline \multirow[t]{3}{*}{2} & \multirow[t]{3}{*}{58} & \multirow[t]{3}{*}{$\mathrm{M}$} & Bilat LL weakness & 3 yrs \\
\hline & & & Bilat LL paresthesia & 2 yrs \\
\hline & & & Erectile dysfunction \& urinary retention & $1 \mathrm{yr}$ \\
\hline \multirow[t]{2}{*}{3} & \multirow[t]{2}{*}{56} & \multirow[t]{2}{*}{ M } & LL paresthesia \& weakness & $3 \mathrm{mos}$ \\
\hline & & & Bowel \& bladder involvement & $1 \mathrm{wk}$ \\
\hline \multirow[t]{3}{*}{4} & \multirow[t]{3}{*}{78} & \multirow[t]{3}{*}{ M } & Bilat LL weakness & $1 \mathrm{yr}$ \\
\hline & & & Bilat LL numbness & $1 \mathrm{yr}$ \\
\hline & & & Bowel \& bladder involvement & $1 \mathrm{wk}$ \\
\hline \multirow[t]{3}{*}{5} & \multirow[t]{3}{*}{53} & \multirow[t]{3}{*}{ M } & $\begin{array}{l}\text { Bilat LL weakness \& reduced sensa- } \\
\text { tions }\end{array}$ & $2.5 \mathrm{yrs}$ \\
\hline & & & Bowel \& bladder involvement & 2 yrs \\
\hline & & & Bilat UL weakness & $8 \mathrm{mos}$ \\
\hline \multirow[t]{3}{*}{6} & \multirow[t]{3}{*}{53} & \multirow[t]{3}{*}{ M } & Bilat LL weakness & \multirow[t]{3}{*}{2 wks } \\
\hline & & & Bilat $L L$ reduced sensations & \\
\hline & & & Bowel \& bladder involvement & \\
\hline \multirow[t]{3}{*}{7} & \multirow[t]{3}{*}{52} & \multirow[t]{3}{*}{ M } & Bilat LL weakness & \multirow[t]{3}{*}{$1 \mathrm{yr}$} \\
\hline & & & Bilat LL reduced sensations & \\
\hline & & & Bowel \& bladder involvement & \\
\hline \multirow[t]{3}{*}{8} & 68 & M & Bilat LL weakness & 2 mos \\
\hline & & & Bilat LL reduced sensations & $2 \mathrm{mos}$ \\
\hline & & & Bowel \& bladder involvement & $1 \mathrm{wk}$ \\
\hline 9 & 67 & M & Bilat LL reduced sensations & $3 \mathrm{mos}$ \\
\hline & & & Bilat LL weakness & $2 \mathrm{mos}$ \\
\hline & & & Bowel \& bladder involvement & $1 \mathrm{wk}$ \\
\hline 10 & 65 & M & Bilat LL weakness & $1 \mathrm{yr}$ \\
\hline & & & Bilat LL reduced paresthesia & $9 \mathrm{mos}$ \\
\hline & & & Bowel \& bladder involvement & 2 wks \\
\hline 11 & 42 & M & Bilat LL weakness & $9 \mathrm{mos}$ \\
\hline & & & Bowel \& bladder involvement & $6 \mathrm{mos}$ \\
\hline & & & Bilat LL numbness & $5 \mathrm{mos}$ \\
\hline 12 & 65 & M & Bilat LL reduced sensations & $1.5 \mathrm{yrs}$ \\
\hline & & & Bilat LL weakness & $1 \mathrm{yr}$ \\
\hline & & & Bladder involvement & $8 \mathrm{mos}$ \\
\hline 13 & 68 & M & Bilat LL reduced paresthesia & $1 \mathrm{mo}$ \\
\hline & & & Bilat LL weakness & \\
\hline & & & Bowel \& bladder involvement & \\
\hline 14 & 42 & M & Bilat LL weakness & $5 \mathrm{mos}$ \\
\hline & & & Bilat LL numbness & 4 mos \\
\hline & & & Bowel \& bladder involvement & $1 \mathrm{mo}$ \\
\hline 15 & 51 & M & Bilat LL weakness & $5 \mathrm{mos}$ \\
\hline & & & Bilat LL reduced sensations & 4 mos \\
\hline & & & $\begin{array}{l}\text { Bowel \& bladder involvement, erectile } \\
\text { dysfunction }\end{array}$ & 3 mos \\
\hline 16 & 72 & $\mathrm{~F}$ & LL weakness & $2 \mathrm{yrs}$ \\
\hline
\end{tabular}

$\mathrm{LL}=$ lower limb; $\mathrm{UL}=$ upper limb riod from January 2007 to January 2013. The demographic characteristics of these patients are presented in Table 1. The level and side of fistula were accurately predicted by Observers 1 and 2 in $81 \%$ and $88 \%$ of cases, respectively. The accuracy improved to $94 \%$ for both observers when localization was made to 1 vertebral level above or below the site of fistula. Among the falsely predicted levels, the side of fistula was correctly identified in one-third and one-half of situations by Observer 1 and Observer 2, respectively. In 1 patient, the level was predicted falsely to be in the mid-thoracic region, whereas DSA confirmed that the meningeal branch of the vertebral artery was the feeder of the fistula.

The interobserver MR reading and observer agreement with gold-standard DSA was found to be highly significant $(p<0.0001)$. Also, the correlation between the side of fistula detected by MRI and DSA was found to be significant $(\mathrm{p}<0.0001)$. The observations are presented in Tables 2-4.

\section{Illustrative Case \\ Case 8}

A 68-year-old man presented with insidious-onset weakness of both lower limbs for 2 months. He also complained of bladder incontinence necessitating continuous bladder drainage, as well as constipation. On clinical examination, he had bilateral paraparesis (the left side was more affected than the right side), absent knee and ankle jerks, and reduced dorsal and lateral column sensations below the knee joint. His general and systemic examinations were unremarkable. The MRI examination demonstrated cord hyperintensity over the lower dorsal cord and conus, with prominent intradural perimedullary flow voids. The analysis of 3D SPACE MRI suggested a possible fistula site at right $\mathrm{T}-12$, which was confirmed by limited spinal angiogram (Figs. 1 and 2). The SDAVF was completely embolized using 33\% Histoacryl glue, achieving complete obliteration of fistula. He showed significant improvement in neurological status in the next 48 hours and had nearcomplete recovery of the lower limb motor power as well as bowel and bladder functions at 6-month follow-up.

\section{Discussion}

Spinal dural fistula is characterized by abnormal communication between the radiculomeningeal artery and the radicular vein located at the dorsal surface of the dural root sleeve in the intervertebral foramen. The fistulous communication causes arterialization of veins and elevation of venous pressure, which impedes the normal venous drainage of the spinal cord and results in cord venous hypertension. The symptoms may be nonspecific

TABLE 2. Observer findings in identification of the site of fistula

\begin{tabular}{ccc}
\hline Observer & Localization by MRI & Localization to 1 Vertebral Level \\
\hline 1 & $13 / 16 \mathrm{pts}(81 \%)$ & $15 / 16 \mathrm{pts}(94 \%)$ \\
\hline 2 & $14 / 16 \mathrm{pts}(88 \%)$ & $15 / 16 \mathrm{pts}(94 \%)$ \\
\hline
\end{tabular}

Pts $=$ patients 
TABLE 3. Observer findings in identification of the side of fistula

\begin{tabular}{ccc}
\hline Observer & Localization by Side & $\begin{array}{c}\text { Accurate Side Localization at } \\
\text { Erroneously Predicted Level }\end{array}$ \\
\hline 1 & $14 / 16 \mathrm{pts}(88 \%)$ & $1 / 3 \mathrm{pts}(33 \%)$ \\
\hline 2 & $15 / 16 \mathrm{pts}(94 \%)$ & $1 / 2 \mathrm{pts}(50 \%)$ \\
\hline
\end{tabular}

initially and may often mimic other common neurological disorders such as neuropathy, radiculopathy, or spinal canal stenosis. The disease relentlessly progresses to irreversible neurological sequelae if unrecognized early in the course of illness. The disease is curable, and neurological changes can be reversed completely following the successful treatment of fistula. This highlights the importance of precise diagnosis by noninvasive modalities such as MRI, so that even patients with low probability of fistula can be evaluated reliably while avoiding unnecessary invasive catheter angiography. $8,10,12$

The MRI technique has been shown to be highly sensitive and specific in the diagnosis of SDAVF. ${ }^{22}$ The MRI findings of SDAVF include the presence of flow voids, cord hyperintensity, cord expansion, or cord enhancement. The presence of cord hyperintensity or flow voids had $100 \%$ sensitivity and $97 \%$ specificity for the diagnosis of fistula. The absence of these 2 findings is reported to have $100 \%$ negative predictive value. ${ }^{22} \mathrm{~A}$ more specific finding is the presence of flow voids; however, this may not be evident in $20 \%$ of patients. Catheter angiography remains the gold-standard test to confirm the diagnosis and to locate the level of SDAVF. ${ }^{13}$ Angiography can be time-consuming and at times frustrating when the angiographer is unable to cannulate the segmental arteries, especially when the aorta is atherosclerotic and tortuous. A prolonged procedure adds discomfort for the patient, and exposes patients to higher radiation, contrast overload, and the risk of nephrotoxicity.

Over the last decade, several MRI, MRA and CTA techniques have been investigated to localize the feeding arteries of SDAVF. Initial MR techniques, such as 2D or 3D phase-contrast MRA and 3D time-of-flight MRA, were able to detect and localize the fistula in more than two-thirds of patients. However, these techniques had several limitations, such as incomplete visualizations of the draining vein or feeder, need for accurate timing to avoid venous contamination and tissue contrast uptake, and deduction of the site of fistula by indirect methods, which localized the level to 2 or 3 vertebral levels. ${ }^{4}$

A larger study using a similar contrast-enhanced MR technique reported an accuracy of fistula site detection of $50 \%$, which improved to $73 \%$ for localization within 1 ver- tebral level. ${ }^{20}$ The fast 3D contrast-enhanced MRA technique used ultra-short echo time to reduce the acquisition time to less than 1 minute, which permitted the capture of contrast in the different phases of circulation. With this technique, accurate localization of fistula was achieved in all patients with spinal arteriovenous malformations and in 2 of 3 patients with SDAVF. 5

Later studies reported the usefulness of centric-ordered contrast-enhanced MRA in the detection and localization of spinal vascular malformations.,15,17 The technique involved rapid bolus injection of contrast agent followed by automatic detection of contrast arrival, which triggered acquisition of fast 3D gradient-echo MR angiographic sequences with centric-ordered phase encoding. The above technique was improved with a larger FOV and a higher gadolinium dose to improve the visualization of normal and abnormal arteries, capture different phases of vascular contrast enhancement, study a larger area of interest, and thus enable the radiologist to complete the MR examination in a single session. ${ }^{17}$

Luetmer et al. identified the site of fistula in only $60 \%$ of patients (13 of 22), although SDAVF was accurately diagnosed in $90 \%$ of their patients (20 of 22), primarily due to inadequate coverage of MR angiography. ${ }^{15}$ Using a similar technique with a larger FOV of $50 \mathrm{~cm}$, Mull et al. were able to localize the feeder accurately in $73 \%$ of patients (14 of 19) and within 1 vertebral level in all of the patients. ${ }^{17}$ Farb et al. demonstrated the site of fistula in $45 \%$ of patients (4 of 9), which improved to $88 \%$ (8 of 9 patients) with additional second or third MRA covering additional regions of the spine. ${ }^{7}$ Applying a similar protocol in a larger cohort, Lindenholz et al. reported accurate identification of the site of dural fistula in $81 \%$ of cases; however, the site could not be determined in $8 \%$ of the fistulas. ${ }^{14}$

The time-resolved MRA technique is increasingly being used to noninvasively study the vascular structures and has also shown improved performance in the detection and localization of SDAVF.,2,19 This technique involves preferential rapid sampling of the central k-space region, which is updated continuously to provide a series of timeresolved images of different phases of angiograms. Using this technique, Amarouche et al. examined 33 patients with SDAVF and accurately determined the level of fistula in $70.2 \%$ of patients, which improved to $82 \%$ when the fistula was considered within 1 vertebral level. ${ }^{2}$ In a similar study, the level of fistula was localized to 1 vertebral level in $85 \%$ of patients with SDAVF, although accurate identification was only possible in less than $50 \%$ of the cases. ${ }^{19}$ Compared with contrast-enhanced MR techniques, there are fewer reports on noncontrast techniques for the as-

\begin{tabular}{|c|c|c|c|c|}
\hline & \multicolumn{2}{|c|}{ Vertebral Localization of Fistula } & \multicolumn{2}{|c|}{ Side of Fistula } \\
\hline & Observer 1 & DSA & Observer 1 & DSA \\
\hline Observer 1 & NA & $0.892(0.0001)$ & NA & $0.872(0.0001)$ \\
\hline Observer 2 & $0.848(0.0001)$ & $0.990(0.0001)$ & $0.862(0.0001)$ & 1.00 \\
\hline
\end{tabular}



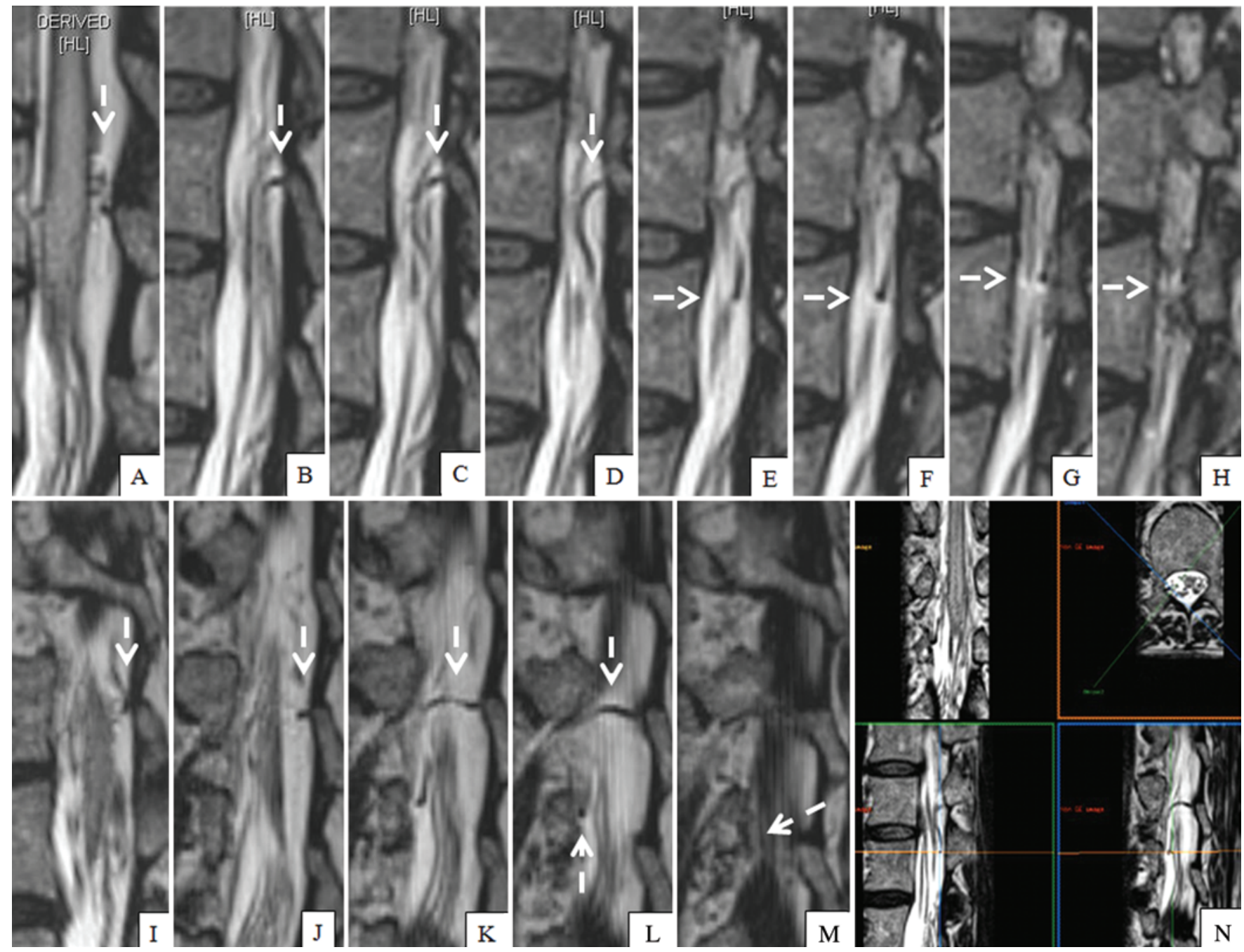

FIG. 1. Midline 3D SPACE MRI showed multiple tortuous dilated veins (white arrow in A), which are followed in oblique sagittal (B-H) and oblique coronal (I-M) planes to the pedicle of the T-12 vertebral body. The radicular vein is demonstrated by white arrows in images B-M. The appropriate plane depicting entry of the vein into the foramen is obtained via the oblique projections, the plane of which is decided from the orthogonal planes. An example of such a plane is shown in panel $\mathbf{N}$. Figure is available in color online only.

sessment and localization of the fistula. Volumetric MR sequences, such as 3D CISS (constructive interference in steady state) and phase-cycled FIESTA (fast imaging employing steady-state acquisition), are reported to be useful in the diagnosis and localization of the SDAVF. ${ }^{16}$ The authors could accurately localize the level of fistula in 3 patients studied. These sequences have high spatial resolution and excellent signal-to-noise ratio, which allowed visualization of smaller vascular and neural structures.

There are only a few studies reported in the literature demonstrating the usefulness of multislice CT angiographic studies in the evaluation and localization of SDAVF. ${ }^{10,21,23}$ The CT study identifies the location of the fistula as focal enhancement of the nerve root, and dural fistula is identified as the presence of dilated perimedullary veins in the spinal canal. These reports showed that the level could be accurately depicted in more than $75 \%$ of the patients with SDAVF. The ability to depict the site of fistula depended on the detector size of the CT scanner, amount of contrast injected, scan parameters, and coverage area studied. ${ }^{23}$ Large-detector CT covers a large FOV and shortens the scan time. However, it may capture the contrast in the arterial phase without adequate opacification of draining veins, and thus may require a second run to adequately visualize the intradural veins. Single or multiple CT runs also raise the concern of increased radiation exposure. Additionally, the ability of CT to locate the side or multifocality of SDAVF remains unknown. ${ }^{23}$ A recent study comparing the performance of 64-slice CT and 3-T dynamic contrast-enhanced MR angiography showed good interobserver agreement and intermodality agreement for MRI, whereas it was only moderate for CTA. The fistula localization was accurate in $83 \%$ of cases after consensus reading, whereas it was only $58 \%$ accurate with $\mathrm{CT}$ angiography. Interobserver agreement was noted to be good in both situations. ${ }^{18}$

The technique described in the present study has several advantages. ${ }^{3}$ The 3D SPACE sequence has high spa- 


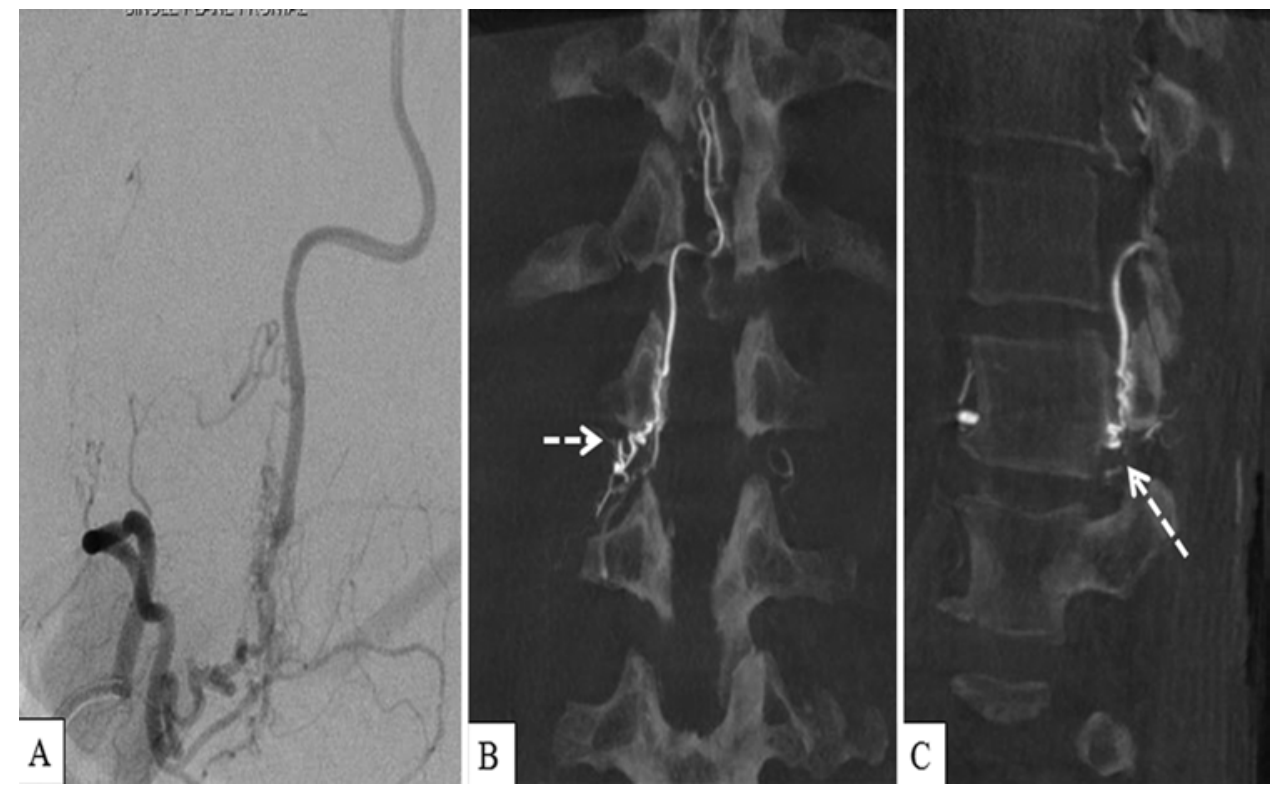

FIG. 2. The diagnostic intercostal angiogram $(A)$ and $3 D$ angiography (B and $C$ ) confirm the diagnosis and location of SDAVF (white arrows in B and C) at the right T-12 level. Note that the course of the radicular vein is similar to what is gleaned from the 3D SPACE MR source images.

tial resolution and superb CSF-tissue contrast. This allows visualization of structures within the spinal CSF space with excellent clarity. The volumetric data acquired are isotropic and allow reconstruction in any plane, achieving high resolution. Additionally, minimal-intensity projection (MIP) images could be obtained, from which angiographic dark-blood images could be reconstructed with thin MIPs. ${ }^{16}$ The scan time is acceptable, and the entire spine study could be completed at 2 stations in 10 minutes. Postprocessing and fusion of the 2 stations to produce a single image could be performed in less than 1 minute. This allowed immediate screening of the study and enabled the operator to decide on further sequences or contrast study for additional evaluation. The T2 contrast of the tissue is better with 3D SPACE than with CISS (constructive interference in steady state) sequences; hence, we have routinely incorporated the 3D SPACE sequence for evaluating spinal pathologies since 2007. Additionally, the entire spine, from foramen magnum to coccyx, can be combined in 2 stages. Thus, the situation of omission of any segment of spine and subsequent reevaluation does not arise.

Compared with contrast-enhanced CTA/MRA and previously described volumetric MR reports, 3D SPACE techniques have distinct advantages. Information is obtained without the administration of contrast and hence there is no risk of nephrotoxicity or nephrogenic systemic fibrosis. The study could be repeated if there were movement artifacts without additional risks, which may not be possible or may necessitate additional gadolinium administration in contrast-based MR techniques. ${ }^{19}$

A second advantage is that the whole spine is covered and studied in 10 minutes. Hence, addition of this sequence to routine MR study results in only mild prolongation of MR examination time (approximately 8 minutes longer compared with contrast-based methods). In contrast-en- hanced MR/time-resolved MR angiography, the coverage is limited (50 cm or less). Therefore, the fistulas located outside the FOV are missed, resulting in a false-negative conclusion. ${ }^{20}$ In fact, most studies have included the midthoracic and lumbar regions in the routine evaluation, leaving out the cervical and lumbosacral areas.

Third, inherent T2-weighted contrast helps to identify signal changes of cord parenchyma; thus, the 2 specific MR findings of SDAVF can be appreciated in the same sequence itself. However, further study is required to validate this observation. Fourth, black-blood angiographic images can be obtained with thick MIPs, complementing noninvasive angiography and helping to focus the confirmatory spinal DSA.

Fifth, the sequence is routinely available from most MR vendors and does not require specific hardware or vendorspecific software. Hence, it can also be performed at most of the peripheral MR centers. Contrast-enhanced/timeresolved MR requires specific hardware and software that may not be widely available on all MR systems. Finally, in time-resolved MRA, spatial and temporal resolution and extent of coverage are interdependent; hence, improving one of the factors can inversely affect the other parameter. The spatial resolution of a high-resolution 3D volumetric T2-weighted MR sequence is comparable to that of timeresolved MR techniques.

Our results are comparable to those of contrast-enhanced MRA as well as time-resolved MRA techniques reported by other investigators. ${ }^{1,2,19} \mathrm{We}$ found good agreement between the 2 observers, achieving more than $80 \%$ accuracy in the correct identification of the fistulous site. Accuracy was improved to more than $90 \%$ when location within 1 vertebral level was considered. These findings are marginally superior to the observations made by other examiners. We believe this is due to superior spatial reso- 
lution of the MR sequence, isotropic volumetric acquisition allowing for multiplanar reconstructions, whole-spine coverage, and a diligent search for fistula.

There are a few disadvantages of this sequence pertaining to SDAVF evaluation. Although the arterial feeder and draining vein can be depicted, the actual fistula site is not demonstrable with this sequence. The superb delineation of vessels is due to superior CST-tissue contrast. Diminution of CSF space in spinal canal stenosis or other pathologies could hamper the localization of fistula. This sequence is not fast compared with contrast-enhanced MR techniques and cannot demonstrate hemodynamic alterations through different phases of circulation. ${ }^{17}$ Additionally, the location of fistula at the site of fusion of 2 stages also could affect optimal visualization, which happened with one of our patients. Inability to detect normal spinal arteries that may impact therapeutic decision making also have to be kept in mind.

The present study has several strengths. It was conducted with readers who were blinded to the spinal angiographic findings, and the performance of the MR sequence was validated between the readers by measuring interobserver variability. Good interrater concordance suggests that the findings could be reproducible among other radiologists. Despite improved localizability, there are important limitations. The investigators were aware of the diagnosis but not the location. This may have biased the readers toward a diligent search for the location of fistula. How this could have negatively impacted the study remains unknown. The presence of flow voids is reported to be very specific for the diagnosis of SDAVF, which could be readily identified with this sequence. ${ }^{5}$ The sensitivity and specificity of the SPACE sequence for SDAVF remain unknown and need to be investigated further in case-control studies. Future studies should focus on this issue, as well as its usefulness in metachronous SDAVF or MR-negative SDAVF.

\section{Conclusions}

Our study demonstrated that volumetric noncontrast 3D SPACE MR sequence is valuable in the precise detection of the site of SDAVF. This sequence is available from most MR vendors and can be incorporated into the routine MR examination with little time delay. The information gathered could help the interventionist plan and perform a focused spinal DSA to confirm the diagnosis and expeditiously treat in the same sitting.

\section{References}

1. Ali S, Cashen TA, Carroll TJ, McComb E, Muzaffar M, Shaibani A, et al: Time-resolved spinal MR angiography: initial clinical experience in the evaluation of spinal arteriovenous shunts. AJNR Am J Neuroradiol 28:1806-1810, 2007

2. Amarouche M, Hart JL, Siddiqui A, Hampton T, Walsh DC: Time-resolved contrast-enhanced MR angiography of spinal vascular malformations. AJNR Am J Neuroradiol 36:417422, 2015

3. Baumert B, Wörtler K, Steffinger D, Schmidt GP, Reiser MF, Baur-Melnyk A: Assessment of the internal craniocervical ligaments with a new magnetic resonance imaging sequence: three-dimensional turbo spin echo with variable flip-angle distribution (SPACE). Magn Reson Imaging 27:954-960, 2009

4. Bowen BC, Fraser K, Kochan JP, Pattany PM, Green BA, Quencer RM: Spinal dural arteriovenous fistulas: evaluation with MR angiography. AJNR Am J Neuroradiol 16:2029_ 2043, 1995

5. Binkert CA, Kollias SS, Valavanis A: Spinal cord vascular disease: characterization with fast three-dimensional contrast-enhanced MR angiography. AJNR Am J Neuroradiol 20:1785-1793, 1999

6. Chen J, Gailloud P: Safety of spinal angiography: complication rate analysis in 302 diagnostic angiograms. Neurology 77:1235-1240, 2011

7. Farb RI, Kim JK, Willinsky RA, Montanera WJ, terBrugge $\mathrm{K}$, Derbyshire JA, et al: Spinal dural arteriovenous fistula localization with a technique of first-pass gadoliniumenhanced MR angiography: initial experience. Radiology 222:843-850, 2002

8. Forbes G, Nichols DA, Jack CR Jr, Ilstrup DM, Kispert DB, Piepgras DG, et al: Complications of spinal cord arteriography: prospective assessment of risk for diagnostic procedures. Radiology 169:479-484, 1988

9. Hartmann J, Rabinstein AA: Can we rule out a spinal arteriovenous fistula using only MRI? Yes, we can. Neurology 79:15-16, 2012

10. Jellema K, Tijssen CC, van Gijn J: Spinal dural arteriovenous fistulas: a congestive myelopathy that initially mimics a peripheral nerve disorder. Brain 129:3150-3164, 2006

11. Kirsch M, Berg-Dammer E, Musahl C, Bäzner H, Kühne D, Henkes H: Endovascular management of spinal dural arteriovenous fistulas in 78 patients. Neuroradiology 55:337-343, 2013

12. Krings T, Geibprasert S: Spinal dural arteriovenous fistulas. AJNR Am J Neuroradiol 30:639-648, 2009

13. Krings T, Lasjaunias PL, Hans FJ, Mull M, Nijenhuis RJ, Alvarez H, et al: Imaging in spinal vascular disease. Neuroimaging Clin N Am 17:57-72, 2007

14. Lindenholz A, TerBrugge KG, van Dijk JM, Farb RI: The accuracy and utility of contrast-enhanced MR angiography for localization of spinal dural arteriovenous fistulas: the Toronto experience. Eur Radiol 24:2885-2894, 2014

15. Luetmer PH, Lane JI, Gilbertson JR, Bernstein MA, Huston J III, Atkinson JL: Preangiographic evaluation of spinal dural arteriovenous fistulas with elliptic centric contrast-enhanced MR Angiography and effect on radiation dose and volume of iodinated contrast material. AJNR Am J Neuroradiol 26:711-718, 2005

16. Morris JM, Kaufmann TJ, Campeau NG, Cloft HJ, Lanzino $\mathrm{G}$ : Volumetric myelographic magnetic resonance imaging to localize difficult-to-find spinal dural arteriovenous fistulas. J Neurosurg Spine 14:398-404, 2011

17. Mull M, Nijenhuis RJ, Backes WH, Krings T, Wilmink JT, Thron A: Value and limitations of contrast-enhanced MR angiography in spinal arteriovenous malformations and dural arteriovenous fistulas. AJNR Am J Neuroradiol 28:1249_ 1258,2007

18. Oda S, Utsunomiya D, Hirai T, Kai Y, Ohmori Y, Shigematsu Y, et al: Comparison of dynamic contrast-enhanced 3T MR and 64-row multidetector CT angiography for the localization of spinal dural arteriovenous fistulas. AJNR Am J Neuroradiol 35:407-412, 2014

19. Saindane AM, Boddu SR, Tong FC, Dehkharghani S, Dion JE: Contrast-enhanced time-resolved MRA for pre-angiographic evaluation of suspected spinal dural arterial venous fistulas. J Neurointerv Surg 7:135-140, 2015

20. Saraf-Lavi E, Bowen BC, Quencer RM, Sklar EML, Holz A, Falcone S, et al: Detection of spinal dural arteriovenous fistulae with MR imaging and contrast-enhanced MR angiogra- 
phy: sensitivity, specificity, and prediction of vertebral level. AJNR Am J Neuroradiol 23:858-867, 2002

21. Si-jia G, Meng-wei Z, Xi-ping L, Yu-shen Z, Jing-hong L, Zhong-hui W, et al: The clinical application studies of CT spinal angiography with 64-detector row spiral CT in diagnosing spinal vascular malformations. Eur J Radiol 71:22 28,2009

22. Toossi S, Josephson SA, Hetts SW, Chin CT, Kralik S, Jun P, et al: Utility of MRI in spinal arteriovenous fistula. Neurology 79:25-30, 2012

23. Yamaguchi S, Nagayama T, Eguchi K, Takeda M, Arita K, Kurisu K: Accuracy and pitfalls of multidetector-row computed tomography in detecting spinal dural arteriovenous fistulas. J Neurosurg Spine 12:243-248, 2010

\section{Disclosures}

The authors report no conflict of interest concerning the materi- als or methods used in this study or the findings specified in this paper.

\section{Author Contributions}

Conception and design: Kannath. Acquisition of data: Alampath, Kannath. Analysis and interpretation of data: Alampath, Kannath. Drafting the article: Alampath, Kannath. Critically revising the article: Kannath, Enakshy Rajan, Thomas. Reviewed submitted version of manuscript: Alampath, Kannath, Thomas, Tirur Raman. Approved the final version of the manuscript on behalf of all authors: Alampath. Statistical analysis: Alampath, Kannath, Sarma. Administrative/technical/material support: Kannath, Enakshy Rajan, Tirur Raman. Study supervision: Kannath, Enakshy Rajan, Thomas.

\section{Correspondence}

Praveen Alampath, Department of Imaging Sciences and Interventional Radiology, Sree Chitra Tirunal Institute of Medical Sciences and Technology, Medical College P.O., Trivandrum, Kerala 695011, India. email: praveen13@outlook.com. 